\title{
Predicting drug pharmacokinetics and effect in vascularized tumors using computer simulation
}

\author{
John P. Sinek · Sandeep Sanga • \\ Xiaoming Zheng • Hermann B. Frieboes • \\ Mauro Ferrari · Vittorio Cristini
}

Received: 23 February 2007 / Revised: 30 January 2008 / Published online: 10 September 2008

C The Author(s) 2008. This article is published with open access at Springerlink.com

\begin{abstract}
In this paper, we investigate the pharmacokinetics and effect of doxorubicin and cisplatin in vascularized tumors through two-dimensional simulations. We take
\end{abstract}

Financial Support from National Cancer Institute, National Science Foundation, and Department of Defense.

J. P. Sinek $\cdot$ X. Zheng $\cdot$ V. Cristini

Department of Mathematics, University of California, Irvine, CA, USA

S. Sanga $\cdot$ V. Cristini

Department of Biomedical Engineering, University of Texas, Austin, TX, USA

S. Sanga $\cdot$ H. B. Frieboes $\cdot$ V. Cristini

Department of Biomedical Engineering, University of California, Irvine, CA, USA

H. B. Frieboes · V. Cristini $(\varangle)$

School of Health Information Sciences, University of Texas Health Science Center, 7000 Fannin, Suite 850A, Houston, TX, 77030, USA

e-mail: Vittorio.Cristini@uth.tmc.edu

M. Ferrari

Center for Nanomedicine, Brown Foundation Institute of Molecular Medicine,

Department of Biomedical Engineering, University of Texas Health Science Center at Houston, Houston, TX, USA

M. Ferrari

Department of Experimental Therapeutics, University of Texas MD Anderson Cancer Center, Houston, TX, USA

M. Ferrari

Department of Bioengineering, Rice University, Houston, TX, USA

V. Cristini

Department of Systems Biology, University of Texas MD Anderson Cancer Center, Houston, TX, USA 
into account especially vascular and morphological heterogeneity as well as cellular and lesion-level pharmacokinetic determinants like P-glycoprotein (Pgp) efflux and cell density. To do this we construct a multi-compartment PKPD model calibrated from published experimental data and simulate 2-h bolus administrations followed by 18-h drug washout. Our results show that lesion-scale drug and nutrient distribution may significantly impact therapeutic efficacy and should be considered as carefully as genetic determinants modulating, for example, the production of multidrug-resistance protein or topoisomerase II. We visualize and rigorously quantify distributions of nutrient, drug, and resulting cell inhibition. A main result is the existence of significant heterogeneity in all three, yielding poor inhibition in a large fraction of the lesion, and commensurately increased serum drug concentration necessary for an average $50 \%$ inhibition throughout the lesion (the $\mathrm{IC}_{50}$ concentration). For doxorubicin the effect of hypoxia and hypoglycemia ("nutrient effect") is isolated and shown to further increase cell inhibition heterogeneity and double the $\mathrm{IC}_{50}$, both undesirable. We also show how the therapeutic effectiveness of doxorubicin penetration therapy depends upon other determinants affecting drug distribution, such as cellular efflux and density, offering some insight into the conditions under which otherwise promising therapies may fail and, more importantly, when they will succeed. Cisplatin is used as a contrast to doxorubicin since both published experimental data and our simulations indicate its lesion distribution is more uniform than that of doxorubicin. Because of this some of the complexity in predicting its therapeutic efficacy is mitigated. Using this advantage, we show results suggesting that in vitro monolayer assays using this drug may more accurately predict in vivo performance than for drugs like doxorubicin. The nonlinear interaction among various determinants representing cell and lesion phenotype as well as therapeutic strategies is a unifying theme of our results. Throughout it can be appreciated that macroscopic environmental conditions, notably drug and nutrient distributions, give rise to considerable variation in lesion response, hence clinical resistance. Moreover, the synergy or antagonism of combined therapeutic strategies depends heavily upon this environment.

Keywords In silico $\cdot$ Simulation $\cdot$ Pharmacokinetics $\cdot$ Prediction $\cdot$ Therapy

Mathematics Subject Classification (2000) $92 \mathrm{C} 45$

\section{Introduction}

While drug resistance to solid tumors is often a consequence of genetic factors, such as upregulation of anti-apoptotic proteins or overexpression of efflux mechanisms, factors at coarser physiological scales may also profoundly influence tumor therapeutic response [50]. A tumor is a heterogeneous three-dimensional composite of fibrous and connective tissues, stromal components, vasculature, and multiple clones of cancer cells. Atop this intrinsic heterogeneity is layered the anatomical and functional irregularity of tumoral vasculature, characterized by erratic flow, collapsed vessels, diminished oxygen tension, and a large mean tissue-to-vessel distance [5,29,30,34, 54]. As a consequence, the tumor microenvironment is highly variable, marked by 
gradients of nutrient and oxygen, resulting in regions of hypoxia, acidity, and necrosis, and heterogeneous proliferation. In order for an anticancer agent to be effective, it must extravasate, diffuse through lesion tissue, and be transported into cells, where it must bind to its target and induce cell apoptosis or mitotic inhibition. The tumor environment is not conducive to these processes [50]. The vessel bed's blood flow and spatial distribution hinder uniform extravasation, calling into question the capability of drug molecules to adequately distribute throughout tissue. Recent experiments in vitro $[38,73,81]$ and in vivo $[40,57]$ demonstrate limited drug penetration through tumors, especially highly protein-bound molecules like doxorubicin and paclitaxel. Once a drug molecule has traversed lesion tissue from its point of extravasation and is presented to a cancer cell, the path from extracellular space to intracellular target is fraught with difficulties ranging from protonation due to the acidic environment, which renders anthracyclines incapable of traversing membrane, to intracellular removal by drug efflux pumps, to cellular processes that effect DNA repair and drug clearance $[3,15,31,65,71,79]$. In addition to pharmacokinetics, drug pharmacodynamics is equally impaired. Significant hypoxia and hypoglycemia throughout may induce cell quiescence, reducing the efficacy of cell-cycle chemotherapeutic agents like doxorubicin and cisplatin $[18,19]$. Hypoglycemia causes the glucose-regulated stress response detrimental to the action of topoisomerase II-directed drugs like doxorubicin $[41,49,64,68]$.

The heterogeneity and three-dimensionality of the tumoral environment presents a challenge to drug assessment, both during development and in the clinic. Whereas a particular drug may show marked activity against a particular specimen in vitro, its potency may vanish or become far less reliable in vivo. This is evidenced by the differential between positive predictive accuracy of in vitro-assisted therapy selection (around 70\%) and negative predictive accuracy (around 90\%), a situation not remarkably changed over the years [26,27]. Supraoptimal delivery of drug to cultured cells eliminates the gauntlet of biobarriers in vivo described above, precluding the variability they induce. A drug that consistently works in vitro can therefore be expected to only sometimes work in vivo. Unraveling the myriad interactions of therapeutic determinants within the complex three-dimensional tumoral environment is evidently difficult, resulting in high costs of drug development and patient suffering.

Perhaps the crystal ball we are attempting to build is incomplete when made only of glass typically found in experimental labs; computer (in silico) simulations based on mathematical modeling and calibrated with experimental data might fulfill a key aspect of the lens. A significant capability of in silico experimentation (including simulated assays) is the complete control over and monitoring of the simulated in vivo tumor environment. Moreover, computer modeling can create hypothetical environments and conditions impossible to achieve otherwise, the study of which is nonetheless instrumental in unraveling disease and drug mechanisms. This expansive control, founded upon an adequately mechanistic mathematical basis, could facilitate the discovery of hypotheses as to why certain drugs or therapeutic strategies would or would not be effective, potentially on a patient-by-patient basis. The relative ease and cost-efficiency of performing simulations could furthermore enable a thorough investigation of strategies, revealing the optimal among them. The judicious combination of this burgeoning technology with the capabilities of the wet-lab is an attractive development in both 
drug discovery and the clinical management of cancer leading to the easing of patients' burdens.

The past two decades have witnessed explosive growth in the mathematical and computational modeling of vascular and avascular tumors $[1,4,7-9,13,36,43,44,56$, 82]. As the basic elements of tumoral growth models have matured, specialized treatments of angiogenesis and flow [2,46,47,69], drug delivery and response [33,40,52, $66,80]$, and effects of the tumoral microenvironment $[23,45]$ have been developed. Some simulation and analysis has provided great insight using one-dimensional modeling (employing cylindrical or spherical symmetry) without discrete vasculature. More recently, powerful numerical methods have been developed so as to simulate multidimensional complex morphological progression and its relation to cell phenotype and the microenvironment involving, for example, nutrient and biomechanical tissue response [23,45,82].

In the present paper we examine therapeutic efficacy of two common drugs, cisplatin and doxorubicin, in relation to phenotypic and microenvironmental conditions. Model parameters govern extracellular drug/tissue diffusivity; cellular uptake, efflux, and metabolism; cellular density; and the effect of nutrient heterogeneity on drug action. Simulations are performed in a two-dimensional (non-symmetric) setting employing discrete vasculature, which enables the incorporation of morphological and topological influence on drug and nutrient distributions. The effect of these distributions on therapeutic efficacy is of special interest. Sinek et al. [66] had earlier performed a similar investigation; however, the pharmacokinetics and pharmacodynamics (PKPD) component was rudimentary, assuming one homogenous lesion compartment and not based upon experimentally acquired parameter values. In the present work we implement an extensive multi-compartment PKPD component whose parameter values are calibrated via published experimental data. This enables a comparison of the tissueand cell-level drug dynamics of the two drugs, and facilitates the generation of hypotheses to explain their in vivo characteristics. We ask that the reader consider that if doxorubicin and cisplatin were discovered only today, the simulations herein could be seen as providing insight into their anticipated in vivo performance, potentially streamlining and reducing costs of development. Indeed, the methodology presented herein could, with additional development, be applied to both established and nascent drugs to the end of refining clinical trials and assisting in clinical therapeutic strategy to improve patient comfort and survival.

\section{Mathematical model and parameters}

\subsection{Model description}

The multiscale tumor growth and angiogenesis simulator developed by Zheng et al. [82] is used to grow the lesions upon which we simulate chemotherapy. This is a nonlinear, continuum scale, two-dimensional growth engine whose accuracy is made possible by an adaptive finite element mesh due to Cristini et al. [11]. The mesh enables multi-scale computation for finely resolving tumor morphology, especially around important areas such as the necrotic/tumor and tumor/host interfaces and around capil- 
lary sprouts. Realistic and heterogenous vasculature through which nutrient and drug is provided is generated via the angiogenesis model of Anderson and Chaplain [2]. The simulation field incorporates three phases (see Fig. 2): viable cancerous tissue, normal host tissue, and necrotic debris. The lesion/host interface is demarked by thick black contours, while the microvasculature appears as a web of thin red curves. Dark interior regions are necrotic debris.

Briefly, nutrient and oxygen are provided through the discrete microvasculature, which is generated in response to angiogenic regulators produced from perinecrotic cells. This results in proliferation and tumor growth. The simple steady-state diffusion equation

$$
0=k_{\mathrm{v}}(1-n) \delta+D_{n} \nabla^{2} n-k_{n} n
$$

is used to model nutrient delivery and uptake, where $n$ is the local nutrient normalized by the intravascular level, $k_{\mathrm{V}}$ is a measure of vascular porosity ( 0 is impermeable, $\infty$ is completely porous), $\delta$ is the Dirac delta function located along the vasculature, $D_{n}$ is nutrient diffusivity, and $k_{n}$ is the local rate of consumption by cells [82]. The characteristically high porosity of tumor vasculature implies a very high setting of $k_{\mathrm{v}}$ so that, essentially, vasculature provides a constant boundary condition of 1. Experiments given in [51] demonstrate that oxygen penetrates approximately $150 \mu \mathrm{m}$ into in vitro spheroids before falling to about $10 \%$ of serum level. At this point necrosis ensues. Combining this with a diffusivity $D_{n}$ of around $60,000 \mu \mathrm{m}^{2} \mathrm{~min}^{-1}[53,70]$, the nutrient uptake rate is calculated to be $k_{n}=24 \mathrm{~min}^{-1}$. Waste resulting from necrotic cell degradation is assumed to be removed via convection towards and through the tumorhost interface as well as via scavenger cell phagocytosis. In regions where nutrient is sufficient to maintain viability, mitosis is assumed to be directly proportional to its concentration, with the proportionality constant dependent upon the average cell cycle time of the malignant population.

Once the tumors are grown, drug administration via the vasculature is simulated by our multi-compartment pharmacokinetics model, based upon earlier work of $[15,17$, 20,21]. For cisplatin, there are three compartments corresponding to (1) extracellular, (2) cytosolic, and (3) DNA-bound drug. For doxorubicin, there is a fourth compartment corresponding to intracellular organelles, e.g., lysosomes. The system of equations governing transport for both drugs (with different parameter values) is

$$
\begin{aligned}
& \dot{s}_{1}=k_{\mathrm{v}}\left(s_{\mathrm{v}}-s_{1}\right) \delta+D_{s} \nabla^{2} s_{1}-k_{12}^{\prime} s_{1}+k_{21}^{\prime}\left(s_{2} / 10^{6} V_{\mathrm{c}}\right) \\
& \dot{s}_{2}=k_{12} 10^{6} V_{\mathrm{c}} s_{1}-k_{21} s_{2}+k_{32} s_{3}-k_{23} s_{2}\left(1-s_{3} / s_{\mathrm{m}}\right)+k_{42} s_{4}-k_{24} s_{2} \\
& \dot{s}_{3}=k_{23} s_{2}\left(1-s_{3} / s_{\mathrm{m}}\right)-k_{32} s_{3}-k_{3} s_{3} \\
& \dot{s}_{4}=k_{24} s_{2}-k_{42} s_{4}
\end{aligned}
$$

where $s_{i}$ represents drug concentration in compartment $i, k_{i j}$ represents a transfer rate from compartment $i$ to $j$, and $k_{i}$ represents a rate of permanent removal from compartment $i$ and the system. $s_{\mathrm{V}}$ is intravascular drug concentration during bolus, and $s_{\mathrm{m}}$ is a DNA saturation parameter relevant to doxorubicin. $V_{\mathrm{c}}$ is the volume of a cell (assumed spherical with diameter $10 \mu \mathrm{m}$, yielding $V_{\mathrm{c}}=520 \mathrm{fL} \mathrm{cell}^{-1}$ ) and appears in the first 
two equations to reconcile the dimensions of $s_{\mathrm{V}}$ and $s_{1}(\mu \mathrm{M})$ with the dimensions of all other compartments (fmoles/cell). $k_{\mathrm{v}}$ and $\delta$ are the same as in Eq. (1). The primed rates appearing in the first equation are related to their unprimed counterparts via $k_{i j}^{\prime}=k_{i j} / F$ where $F$ is the extracellular fraction of whole tissue. Taking a baseline tumor density of $\rho=1.0 \mathrm{E} 9$ cells $\mathrm{mL}^{-1}$, a well-known representative value, in combination with the cell volume previously quoted results in

$$
\begin{aligned}
F & =1-\rho V_{\mathrm{c}}\left(10^{-12} \mathrm{~mL} \mathrm{fL}^{-1}\right) \\
& =0.48,
\end{aligned}
$$

also a reasonable value. Finally, $D_{s}$ is the diffusivity of the drug through extracellular space.

Both cisplatin and doxorubicin pass through cell membrane according to $k_{12}$ (which includes possible pump and transporter activity, as do all other rates). From there, the drugs may efflux according to $k_{21}$ or may bind to DNA according to $k_{23}$. The kinetics differ from here for the two drugs. Cisplatin may be removed according to the rate $k_{3}$, which destroys the functioning of the drug and repairs the DNA [79]. Doxorubicin, however, has an off rate given by $k_{32}$, and moreover may be sequestered and released by lysosomes according to $k_{24}$ and $k_{42}[3,31,59]$. Although lysosomal flow to membrane and exocytosis of sequestered drug plays a role in some drug resistant cell lines, we are not necessarily modeling drug resistance via this function, and so assume this process to be negligible in accordance with [16]. On the other hand, we are concerned with the quantity of drug lysosomes can sequester, as this contributes to the cellular uptake of drug, and hence, its penetration characteristics.

The pharmacodynamics model consists of the Hill-type equation along the lines of those employed in $[20,21]$

$$
E=\frac{N(n)}{1+A^{-1} x^{-m}}
$$

where $E$ is cell inhibition (1 minus surviving fraction), $x$ is DNA-bound drug-time product (area under the curve, or AUC), and $A$ and $m$ are phenomenologically fit parameters. $N(n)$ is a function of nutrient $n$ ranging from 0 to 1 used to mimic the effect of hypoxia and hypoglycemia. Results with doxorubicin show that cells in deeper layers of spheroids do not respond as well to drug as do cells on the surface, even when intracellular drug levels are taken into account $[18,19]$. Other experiments demonstrate reduced response in monolayer when cells are forced into quiescence due to reduced oxygen [68]. Still others show that hypoglycemia can deplete topoisomerase II, thus reducing the effect of some anthracyclines [64]. These results imply that the response of cells to doxorubicin in vivo might correlate to the local nutrient, a phenomenon which we herein refer to as the "nutrient effect." For our purposes, the exact form of $N$ is not important. For simplicity, we choose $N=n^{p}$, where $p$ is a phenomenological parameter derived from the data of [19], and equals 0.4. Since in our model $n$ is normalized with respect to the intravascular level, it runs from 0 to 1 , and thus so does $N$. Furthermore, at full nutrient levels, $N=1$, and so cell inhibition is maximal. In our simulations, drug pharmacokinetics (Eqs. 2) is allowed to proceed from bolus 
Table 1 A complete summary of baseline pharmacokinetics and pharmacodynamics parameters

\begin{tabular}{|c|c|c|c|}
\hline \multirow[t]{2}{*}{ Parameter } & \multirow[t]{2}{*}{ Description } & \multicolumn{2}{|c|}{ Baseline Value } \\
\hline & & Dox & Cis \\
\hline$V_{\mathrm{c}}$ & Cell volume (fL cell-1) & 520 & 520 \\
\hline$\rho$ & Cell density (cells mL ${ }^{-1}$ ) & $1.0 \mathrm{E} 9$ & $1.0 \mathrm{E} 9$ \\
\hline$F$ & Interstitial fraction & 0.48 & 0.48 \\
\hline$D_{n}$ & Nutrient/ECM diffusivity $\left(\mu \mathrm{m}^{2} \mathrm{~min}^{-1}\right)$ & $60 \mathrm{E} 3$ & $60 \mathrm{E} 3$ \\
\hline$D_{s}$ & Drug/ECM diffusivity $\left(\mu \mathrm{m}^{2} \min ^{-1}\right)$ & $1.0 \mathrm{E} 3$ & $30 \mathrm{E} 3$ \\
\hline$k_{n}$ & Nutrient metabolism $\left(\min ^{-1}\right)$ & 24 & 24 \\
\hline$k_{12}$ & Drug uptake $\left(\min ^{-1}\right)$ & 5.40 & 0.054 \\
\hline$k_{21}$ & Drug efflux $\left(\min ^{-1}\right)$ & 5.40 & $1.56 \mathrm{E}-3$ \\
\hline$k_{23}$ & Drug-DNA binding $\left(\min ^{-1}\right)$ & $8.02 \mathrm{E} 5$ & $3.82 \mathrm{E}-4$ \\
\hline$k_{32}$ & Drug-DNA release $\left(\min ^{-1}\right)$ & $1.80 \mathrm{E} 3$ & 0.0 \\
\hline$k_{3}$ & Drug-DNA repair $\left(\mathrm{min}^{-1}\right)$ & 0.0 & 0.015 \\
\hline$k_{24}$ & Lysosomal sequestration $\left(\mathrm{min}^{-1}\right)$ & 10.0 & 0.0 \\
\hline$k_{42}$ & Lysosomal release $\left(\min ^{-1}\right)$ & 0.07 & 0.0 \\
\hline$s_{\mathrm{m}}$ & Drug-DNA capacity (fmole) & 1.00 & $\infty$ \\
\hline$A$ & Phenomenological PD parameter & 0.188 & 7.75 \\
\hline$m$ & Phenomenological PD parameter & 1.14 & 1.58 \\
\hline$p$ & Nutrient effect parameter & 0.4 & 0.0 \\
\hline
\end{tabular}

Tumor growth and angiogenesis parameters can be found in [82]

initiation to washout $20 \mathrm{~h}$ later. During this time the locally varying DNA-bound AUC is calculated and used to find cell inhibition (Eq. 4).

\subsection{Pharmacokinetics model parameters}

A generally acceptable theoretical setup for performing experiments to measure compartmental concentrations (and therefore to derive the rate constants we are after) is either a suspension or monolayer in an inexhaustible drug-laden medium corresponding to $s_{1}$. Under these conditions, the relevant model consists of the last three equations in Eqs. (2), with $s_{1}$ held constant. We will refer to this model as the modified version of Eqs. (2). All model parameters and values are summarized in Table 1. These will be referred to as the baseline values, some of which will be adjusted later to simulate different tumor characteristics and therapeutic treatments. We emphasize that parameter values, having been derived from a variety of published experimental data spanning many years and cell types, correspond to a prototypical tumor and cancer cells suitable for the simulations herein, but not necessarily representative of any particular clinical specimen.

Cisplatin parameters We begin with cisplatin, setting $k_{24}$ and $k_{42}$ to 0 since we assume only three compartments, and $k_{32}$ to 0 since we assume the repair rate $k_{3}$ is the 
dominant removal rate of DNA-bound drug. $k_{3}$ is next obtained as follows. In experiments performed by Sadowitz et al. [60], adducts per million nucleotides on isolated peripheral blood mononuclear cell DNA fall from 75 to 5 and 185 to 40 in $2 \mathrm{~h}$ in two different experiments. Thus, assuming the exponential repair model $\dot{s}_{3}=k_{3} s_{3}$, we calculate the repair rate to be about $0.015 \mathrm{~min}^{-1}$. An initial estimate of $k_{23}$ is then made as follows. Sadowitz shows that for $7 \mu \mathrm{M}$ cisplatin, in $2 \mathrm{~h}$ peripheral blood mononuclear cells accumulate from about 25 (non-thiol-blocked cells) to 175 (thiolblocked cells) adducts per million nucleotides. Assuming that DNA consists of about 1.25E6 kbp, this converts to from 1.04E-4 to 7.27E-4 fmoles of Pt docked on the DNA (1 atom/adduct). Neglecting the cell membrane and supposing DNA to be exposed directly to the drug, we have the ODE $\dot{s}_{3}=7 \lambda_{23}-k_{3} s_{3}$, where $\lambda_{23}$ is a clearance parameter $\left(\mathrm{fL} \min ^{-1}\right)$. The solution is $s_{3}=7\left(\lambda_{23} / k_{3}\right)\left(1-\exp \left(-k_{3} t\right)\right)$. Substituting values of $k_{3}=0.015 \mathrm{~min}^{-1}, t=120 \mathrm{~min}$, and 1.04E-4 $\leq s_{3} \leq 7.27 \mathrm{E} 0-4$ fmole yields $0.27 \leq \lambda_{23} \leq 1.9 \mathrm{fL} \mathrm{min}^{-1}$. To convert this to a rate we use the relation $k_{23}=\lambda_{23} / V_{\mathrm{c}}$, arriving at $5.19 \mathrm{E}-4 \leq k_{23} \leq 3.65 \mathrm{E}-3 \mathrm{~min}^{-1}$. The assumption that DNA was exposed directly to the cisplatin solution means that this rate is only a bootstrap approximation and must be refined. We note that the extremely low ratio of adducts per kbp implies that the saturation capacity of DNA with respect to cisplatin is never approached, and so set $s_{\mathrm{m}}$ to $\infty$.

Next, we estimate $k_{12}$ and $k_{21}$. While doing this we will refine our initial estimate of $k_{23}$. The whole procedure involves fitting the best curves to data from Troger et al. [76] (Fig. 1a). Troger exposed human tongue carcinoma CAL-27 cells in monolayer to four different concentrations of cisplatin and then measured total intracellular amount of Pt at selected times. This corresponds to $s_{2}+s_{3}$ in our model. Beginning with the previous estimate of $k_{23}$ and setting $s_{1}$ to concentrations used by Troger, we adjust $k_{12}$ and $k_{21}$ in the modified version of Eqs. (2) until a good fit of Troger's data is obtained. Simultaneously, we adjust $k_{23}$ to keep the DNA-bound drug true to results of Sadowitz previously discussed. We remark that the disparity between the inward and outward rates derived for cisplatin may be due in part to carrier-mediated transport, e.g., the CTR1 influx transporter.

Doxorubicin parameters Proceeding to doxorubicin we first obtain an acceptable range for $k_{12}$ and $k_{21}$ from the literature. For a variety of anthracyclines, including doxorubicin, initial estimates of cell membrane permeability $P$ are taken from experiments with SU-4 and SU-4R wildtype and resistant human lymphoma cells [17], from experiments with EHR2 and EHR2/DNR+ wildtype and resistant Ehrlich ascites tumor cells $[15,16]$, and from experiments with MDA-468 breast cancer cells [40]. The range reported is $2.4 \leq P \leq 1000 \mu \mathrm{m} \mathrm{min}^{-1}$. The relation $k_{12}=P A_{\mathrm{c}} / V_{\mathrm{c}}$, where $A_{\mathrm{c}}$ represents the cell membrane area, can then be used to arrive at an initial range of $1.4 \leq k_{12} \leq 600 \mathrm{~min}^{-1}$, which will be refined later. In the case of passive diffusion, $k_{21}=k_{12}$. We note that these values are far larger than those obtained for cisplatin previously. More generally, it has been remarked that cell membrane permeability for cisplatin is much lower than for doxorubicin, etoposide, and vinblastine, although all four drugs are thought to enter cells by passive diffusion [35].

We next turn our attention to DNA-binding affinity. Given the great DNA affinity of the anthracyclines, saturability of the DNA must be taken into account, requiring 


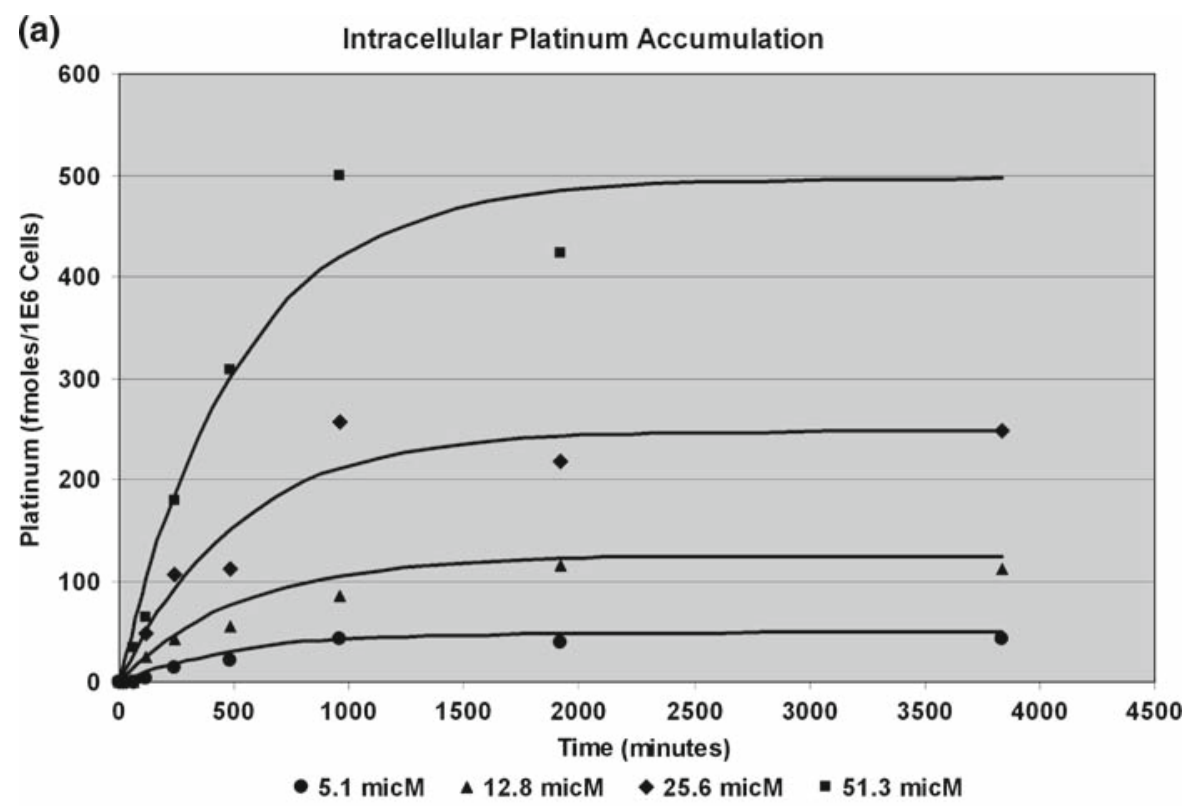

Cell Inhibition for $\mathrm{C}$ is and Dox

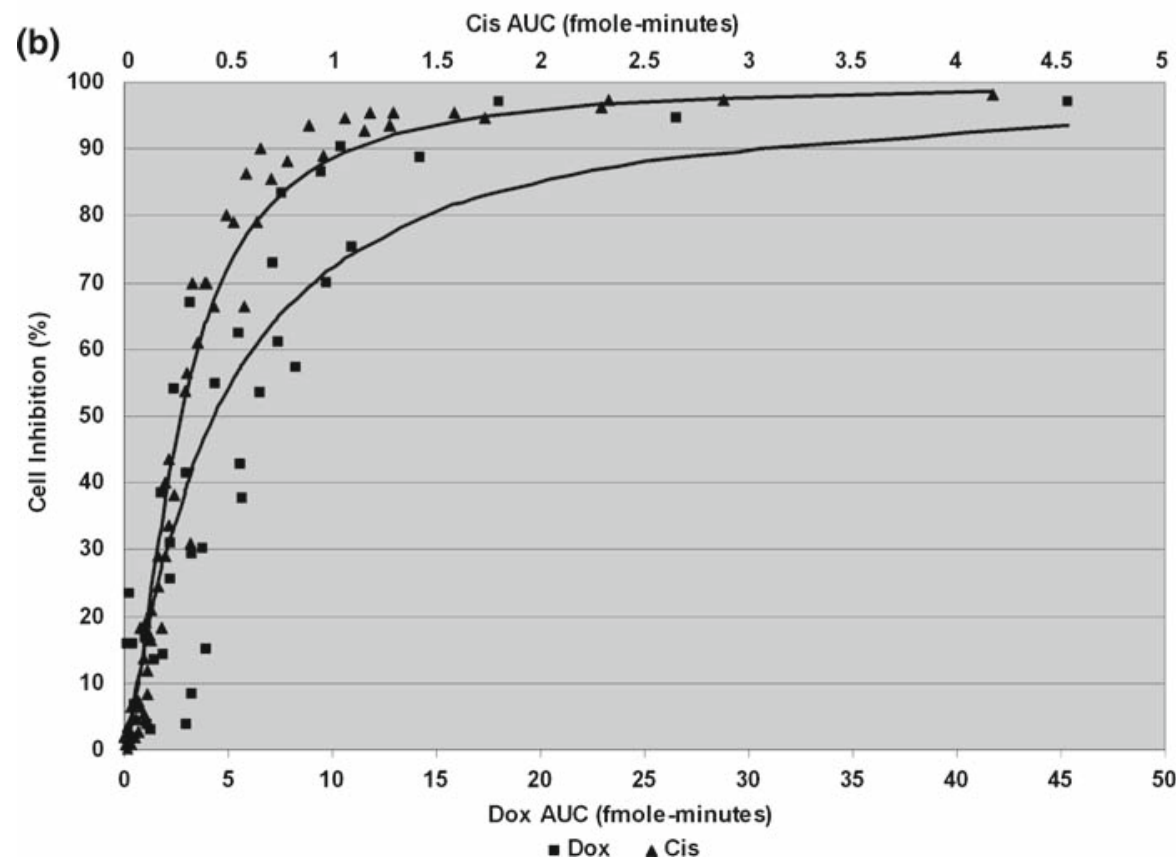

Fig. 1 a Data from Troger et al. [76] used to calibrate $k_{12}, k_{21}$, and $k_{23}$ for the cisplatin model. Parameters are fit simultaneously to all four curves treated as one set of data. b Cell inhibition fits for Eq. (4) using Levasseur's [42] data on A2780 ovarian cancer cells exposed in monolayer 
an estimate of $s_{\mathrm{m}}$. There is evidence a typical anthracycline molecule intercalation occludes from 3 to 10 binding sites in a manner that cannot be corrected exactly by a factor $[48,59,74]$; however, to a first approximation we assume that such a correction can be applied. Demant and Friche [15] report a DNA binding site concentration of about $5 \mathrm{mM}$ within a cell volume of $1000 \mathrm{fL}$, yielding 5 fmoles of sites. A low value of 0.7 fmoles is obtained by using our assumed value of $1.25 \mathrm{E} 6 \mathrm{kbp}$ and the reported site exclusion parameter of about 3 from Rizzo et al. [59]. Tarasiuk et al. [74] find that the DNA of human lymphocytes is comprised of about 6.0E6 kbp and that one intercalating molecule of doxorubicin requires 10 base pairs. Thus, Tarasiuk's data implies a factor-corrected quantity of 1 fmole of binding sites, which we take as a representative value of $s_{\mathrm{m}}$.

DNA binding kinetics of the anthracyclines is nontrivial, perhaps requiring multiple steps and demonstrating sequence specificity [58,59]. Bearing this in mind, as an approximation it will suffice to assume non-specific, one-step binding and unbinding according to the chemical reaction

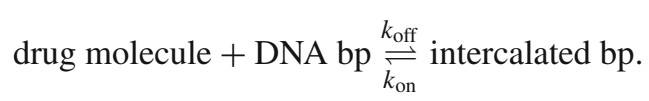

A representative value for the binding coefficient in the above equation for doxorubicin is reported as $k_{\mathrm{on}}=4.2 \mathrm{E} 8 \mathrm{M}^{-1} \mathrm{~min}^{-1}$ and a value of the unbinding coefficient (identical with $k_{32}$ ) as $k_{\text {off }}=1800 \mathrm{~min}^{-1}$ [59]. From $k_{\text {on }}$ we calculate a clearance parameter (as with cisplatin) given as $\lambda_{23}=k_{\mathrm{on}} s_{\mathrm{m}}=4.2 \mathrm{E} 8 \mathrm{fL} \mathrm{min}^{-1}$ (being cautious with the scales of our dimensions). $k_{23}$ can then be calculated as $\lambda_{23} / V_{\mathrm{c}}$, given in Table 1.

We next turn our attention to the rates $k_{24}$ and $k_{42}$ governing lysosomal sequestration. Experiments by Hurwitz et al. [31] using U-937 myeloid leukemia cells and their dox-resistant variant U-A10 show that the ratio of DNA-bound to lysosomally sequestered drug is about 3 (Hurwitz uses daunorubicin, an anthracycline related to doxorubicin). In our modified model equations with all other parameters set as described above, the amount of sequestered drug at equilibrium is dependent only upon the ratio $k_{24} / k_{42}$. This ratio furthermore does not affect the equilibrium quantity of DNA-bound drug. Arbitrarily selecting $k_{24}=1 \mathrm{~min}^{-1}$, we find that the appropriate DNA-bound to lysosomally sequestered ratio is obtained by setting $k_{42}$ to 0.007 . Considering that lysosomal membrane permeability is quite high [16], the lysosomally bound drug must achieve equilibrium quickly, which can be modified by changing $k_{24}$ while keeping the ratio $k_{24} / k_{42}$ constant. We find that increasing $k_{24}$ by a factor of 10 reduces the time required for the system (Eqs. 2) to achieve $95 \%$ of equilibrium value ( $\max _{95}$ ) to about $300 \mathrm{~min}$, below which further increases in $k_{24}$ only reduce this time negligibly. Thus, we set $k_{24}=10$ and $k_{42}=0.07$.

To refine our initial range of $k_{12}$ and $k_{21}$, we use the modified version of Eqs. (2) to compare our simulated monolayer uptake profiles of total intracellular drug with those of DeGregorio et al. [14] using human Ewing's sarcoma and rhabdomyosarcoma cells. At $5.40 \mathrm{~min}^{-1}$ both uptake profiles and equilibrium values compare favorably at three test concentrations. 
Drug-tissue diffusivity The last pharmacokinetics parameter values needed are the diffusivities $D_{s}$ of cisplatin and doxorubicin through tumor interstitium. For molecules of their size (dox M.W. = 544, cis M.W. = 300), diffusivity should be about $30,000 \mu \mathrm{m}^{2} \mathrm{~min}^{-1}[53,70]$. However, doxorubicin faces particularly severe barriers due to binding to extracellular constituents such as hyaluronic acid $[37,38]$, and its diffusivity in some tissues has been estimated to be as low as $1000 \mu \mathrm{m}^{2} \mathrm{~min}^{-1}$ [40], which we take as our baseline value.

\subsection{Pharmacodynamics model parameters}

In order to calibrate the pharmacodynamics model (Eq. 4), we use in vitro data of Levasseur et al. [42] with A2780 ovarian cancer cells exposed in monolayer to both doxorubicin and cisplatin over a range of times and concentrations. We assume the previously discussed modified pharmacokinetics model along with the values derived, and simulate Levassuer's exposures followed by approximately $24 \mathrm{~h}$ of drug washout in drug-free medium ( $s_{1}$ is set to 0 ). During this time, DNA-bound AUC is calculated. These data are then used in conjunction with Levasseur's surviving fraction data to fit the parameters $A$ and $m$ in Eq. (4) using Microsoft Excel (Fig. 1b). During this process, nutrient is assumed plentiful $(n=1)$, thus bypassing the nutrient effect.

\section{Computational simulations}

\subsection{Non-dimensionalization and numerical methods}

Non-dimensionalization of Eqs. 2 is via the length- and time-scales

$$
\mathcal{L}=\sqrt{D_{s} / k_{12}} \quad \mathcal{T}=k_{12}^{-1}
$$

with the compartmental concentrations transformed as

$$
\begin{aligned}
& \bar{s}_{1}=s_{1} / s_{\mathrm{V}} \quad \bar{s}_{2}=s_{2} / 1 \mathrm{E} 6 V_{\mathrm{c}} s_{\mathrm{V}} \quad \bar{s}_{3}=s_{3} / 1 \mathrm{E} 6 V_{\mathrm{c}} s_{\mathrm{V}} \\
& \bar{s}_{4}=s_{4} / 1 \mathrm{E} 6 V_{\mathrm{c}} s_{\mathrm{V}} \quad \bar{s}_{\mathrm{m}}=s_{\mathrm{m}} / 1 \mathrm{E} 6 V_{\mathrm{c}} s_{\mathrm{V}}
\end{aligned}
$$

resulting in

$$
\begin{aligned}
& \dot{\bar{s}}_{1}=\bar{k}_{\mathrm{V}}\left(1-\bar{s}_{1}\right) \delta+\bar{\nabla}^{2} \bar{s}_{1}-\bar{s}_{1} / F+\bar{k}_{21} \bar{s}_{2} / F \\
& \dot{\bar{s}}_{2}=\bar{s}_{1}-\bar{k}_{21} \bar{s}_{2}+\bar{k}_{32} \bar{s}_{3}-\bar{k}_{23} \bar{s}_{2}\left(1-\bar{s}_{3} / \bar{s}_{\mathrm{m}}\right)+\bar{k}_{42} \bar{s}_{4}-\bar{k}_{24} \bar{s}_{2} \\
& \dot{\bar{s}}_{3}=\bar{k}_{23} \bar{s}_{2}\left(1-\bar{s}_{3} / \bar{s}_{\mathrm{m}}\right)-\bar{k}_{32} \bar{s}_{3}-\bar{k}_{3} \bar{s}_{3} \\
& \dot{\bar{s}}_{4}=\bar{k}_{24} \bar{s}_{2}-\bar{k}_{42} \bar{s}_{4}
\end{aligned}
$$

The numerical methods for tumor growth and angiogenesis have been described in detail in Zheng et al. [82]. For the reaction-diffusion equations (Eqs. 2) we first use 
Strang splitting. Then the trapezoidal rule is applied to the reaction part, and the CrankNicolson scheme, to the diffusion part. For a description of these methods, see Tyson et al. [78] and references therein.

\subsection{In silico experiments}

Four in silico experiments are performed in the following manner:

1. Three simulated tumors are grown using Zheng et al.'s model [82]. Each lesion represents one replication of each experiment.

2. The pharmacokinetics model (Eqs. 5) is used to deliver drug to the lesions. In each case we hold the intravascular concentration of drug $s_{\mathrm{V}}$ constant for $2 \mathrm{~h}$, then set it to zero for eighteen more hours to allow washout.

3. DNA-bound AUC is computed by re-dimensionalizing $s_{3}$ and time and integrating using Matlab. The result is then used in the pharmacodynamics model (Eq. 4) to compute cell inhibition. Nutrient (Eq. 1) is relevant when the nutrient effect is employed.

Each lesion is produced based upon the same set of growth and vasculature parameters (see Zheng et al. for a complete description), but randomness in the angiogenesis algorithm and slightly different initial shapes produce different vasculatures and morphologies. It is assumed that a tumor in vivo does not grow or regress appreciably during the 20-h course of the therapy we are attempting to simulate, hence we stop tumor and vascular growth during our in silico therapies. Intravascular concentrations are calibrated in each case to produce a total cellular growth inhibition of 50\%. This concentration is referred to as the $\mathrm{IC}_{50}$. The sharp "square wave" delivery of drug is perhaps a caricature of clinical bolus administration, but it allows for consistent analysis and comparison of results.

Our first set of experiments compares DNA-bound drug AUC distributions of doxorubicin and cisplatin under the baseline conditions in Table 1. We furthermore show the homogenizing effect of doxorubicin retention on final DNA-bound AUC [19]. We next investigate the impact of inhibition heterogeneity on dosing requirements, paying particular attention to the nutrient effect for doxorubicin under baseline conditions and improved penetration by, for example, removing hyaluronic acid $[37,38]$. In our third set of simulations we more deeply investigate the effect of doxorubicin penetration therapies under three circumstances: baseline tumor density, high tumor density, and baseline tumor density with Pgp efflux activity. These are chosen because they demonstrate a spectrum of possibilities due to their effect on cellular drug uptake. High tumor density increases uptake, while Pgp efflux decreases it. In order to simulate increased penetration, we increase $D_{s}$ for doxorubicin from its baseline value to $5000 \mu^{2} \mathrm{~min}^{-1}$ for a moderate increase, and 30,000 for the maximum increase, thus matching the performance of cisplatin. To simulate high tumor density we increase $\rho$ by $50 \%$ to $1.5 \mathrm{E} 9$ cells $\mathrm{ml}^{-1}$. This has the effect of lowering the interstitial fraction $F$ to 0.22 , which in turn increases $k_{12}^{\prime}$ and $k_{21}^{\prime}$ while leaving all other rates unchanged. Pgp efflux is simulated by increasing $k_{21}$ by a factor of 10 , which has the effect of reducing all intracellular compartment concentrations by approximately the same factor. 
This is consistent with results of [10] that show Pgp activity can reduce intracellular concentrations of daunorubicin (an anthracycline related to doxorubicin) by up to a factor of 100. In our fourth and final set of experiments we investigate permeabilization therapy with respect to cisplatin, whereby a detergent, such as digitonin, or electropermeabilization is used to increase the permeability of cell membrane [35,72]. We take an extreme case, increasing the rate constants $k_{12}$ and $k_{21}$ from baseline both by a factor of 100. Note that this does not increase the limiting intracellular or DNA-bound levels of drug attained in simulated monolayer, only the rate at which these come to equilibrium. Thus highly permeabilized, DNA-bound max95 is attained at $3.4 \mathrm{~h}$ of exposure; further permeabilization reduces this negligibly. For comparison, $\max _{95}$ is greater than $27 \mathrm{~h}$ for unpermeabilized cells. This therapy is simulated under both in vivo baseline and very high cell densities achieved by increasing the baseline density $75 \%$ to $1.75 \mathrm{E} 9$ cells $\mathrm{ml}^{-1}$. At this density, the interstitial fraction $F$ drops to a mere 0.08. Both of these are further compared to monolayer results to probe the conditions under which in vitro assays can be used to predict clinical efficacy.

Although all treatments described are duplicated in each of the three in silico tumors, we display only representative plots with appropriate summaries of all data. The nutrient effect is only used where noted.

\subsection{Results}

First experiment We begin by examining DNA-bound AUC distributions at various times in the baseline simulated lesions (each lesion corresponding to a column, I, II, or III), shown in Fig. 2. From top to bottom, the times correspond to 2, 8, 14, and $20 \mathrm{~h}$ post-bolus initiation. Levels are normalized relative to the average AUC within viable lesion for comparison of heterogeneity. Although surrounding host tissue cells uptake and bind with drug differently than cancer cells, we make no distinction in these color plots; however, quantitative analytical results only consider DNA-bound drug within viable lesion. The two left column sequences (Lesions I and II) show doxorubicin AUC, while the rightmost column shows cisplatin. For both Lesions I and II, at $2 \mathrm{~h}$ doxorubicin AUC is seen to be about three times the average (dark red) in the vicinity of the vasculature, and almost 0 (blue) elsewhere. The distribution is only slightly more homogeneous by $8 \mathrm{~h}$. By $14 \mathrm{~h}$ the heterogeneity has lessened, with the peaks close to the vasculature reaching only about 2.2. Finally, at the conclusion of washout $20 \mathrm{~h}$ after bolus initiation, the distribution has become much more homogeneous, with peaks only reaching about 1.7 times the average. In contrast, cisplatin distribution within Lesion III remains extremely homogeneous, right at the average, throughout the entire treatment.

The probability distributions at the bottom, corresponding to AUC at $20 \mathrm{~h}$ postbolus initiation, allow for a more quantitative comparison. The two corresponding to doxorubicin show much heterogeneity relative to cisplatin on the right. Using the leftmost distribution as an example, the average DNA-bound AUC is found to be 6.04 fmole min. $25 \%$ of tumor cells receive less than 1.66 fmole min each, while $25 \%$ of tumor cells receive more than 9.54 fmole min. The remaining $50 \%$ of the tumor cells receive between these two values, a range of 7.88 fmole min. When normalized with 

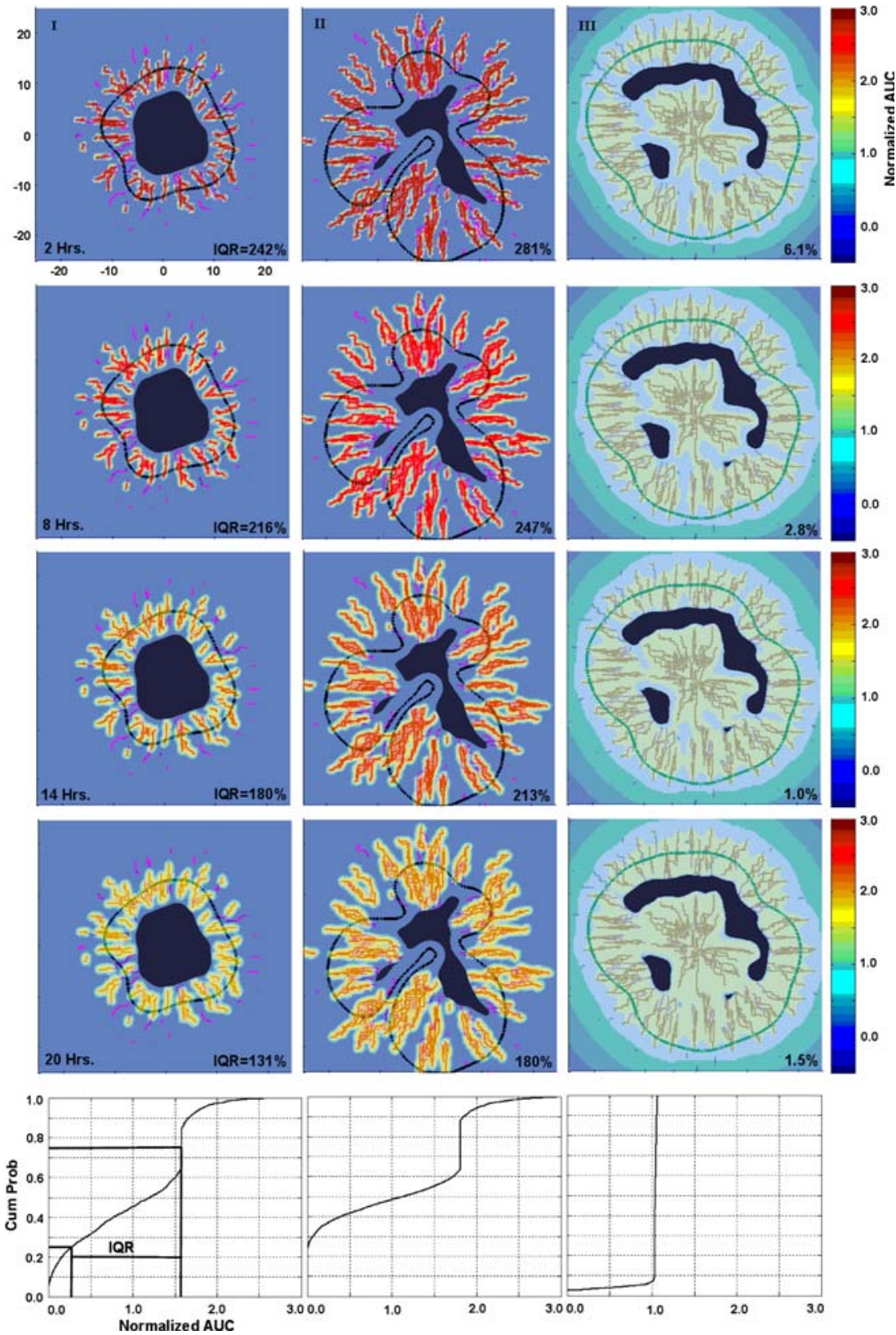

Fig. 2 DNA-bound AUC at four times (rows 2, 8, 14, and $20 \mathrm{~h}$ ) post-bolus initiation for three twodimensional simulated baseline tumor lesions (columns). $I$ and $I I$ are doxorubicin, while III is cisplatin. Results are normalized to average lesion AUC at the time taken to enable comparison of distribution heterogeneities. Thick black contours are tumor boundaries. Thin red curves are vasculature. Dark regions are necrotic areas. Each unit represents $200 \mu \mathrm{m}$. Bottom probability distributions show final AUC distribution at $20 \mathrm{~h}$. A concise measure of heterogeneity is given by the inter-quartile range (IQR), depicted in the lower left graph and explained in the text. Although AUC in host tissue is also shown in plots, analysis considers only DNA-bound drug in viable lesion 
Table 2 Mean \pm SD of the $\mathrm{IC}_{50}$ and the $\operatorname{logs}$ of their ratios with respect to monolayer treatments for experiments to investigate the impact of drug and nutrient heterogeneity

\begin{tabular}{llll}
\hline Nutrient effect & $\mathrm{IC}_{50, \text { mono }}(\mu \mathrm{M})$ & $\mathrm{IC}_{50}(\mu \mathrm{M})$ & $\log \left(\mathrm{IC}_{50} / \mathrm{IC}_{50, \text { mono }}\right)$ \\
\hline Doxorubicin baseline & & & \\
Off & 0.175 & $0.482 \pm 0.163$ & $0.424 \pm 0.138\left(^{*} p<0.05\right)$ \\
On & 0.175 & $1.34 \pm 0.874$ & $0.830 \pm 0.261\left(^{*} p<0.05\right)$ \\
\multicolumn{2}{l}{ Doxorubicin with penetration therapy } & & \\
Off & 0.175 & $0.197 \pm 0.0172$ & $0.0511 \pm 0.0371(p>0.05)$ \\
On & 0.175 & $0.371 \pm 0.0356$ & $0.325 \pm 0.0407\left(^{*} p<0.05\right)$ \\
Cisplatin baseline & & & \\
N/A & 7.05 & $7.14 \pm 0.0757$ & $0.00529 \pm 0.00462(p>0.05)$ \\
\hline
\end{tabular}

$\mathrm{IC}_{50, \text { mono }}$ is the $\mathrm{IC}_{50}$ of baseline cells in monolayer. At the 5\% significance level using a one-tailed $t$ test, the average log ratio for cisplatin does not exceed 0 . On the other hand, in three of the four experiments with doxorubicin, they do. Paired one-tailed $t$ tests show that the average $\log \mathrm{IC}_{50}$ ratios for doxorubicin with the nutrient effect are greater than that without regardless of penetration therapy

respect to the average and expressed as a percent, this yields $131 \%$ (the interquartile range, or IQR), and gives a concise measure of distribution heterogeneity (the closer to 0 , the more homogeneous). IQR's are given at each of the other time points as well. All three tumors, despite varied lesion and vasculature morphologies, demonstrate similar results (not all shown). Doxorubicin AUC IQR's typically lessen from about $250 \%$ at $2 \mathrm{~h}$ to $150 \%$ at $20 \mathrm{~h}$; cisplatin AUC IQR's drop from about 10 to $2 \%$. Interestingly, in the run shown, the heterogeneity for cisplatin increases slightly in the last frame. This happens in one of the other two tumors as well.

Second experiment We next investigate the impact of drug and nutrient heterogeneity on cell inhibition distributions and $\mathrm{IC}_{50}$ 's. Bolus administrations are simulated for cisplatin using baseline lesions exactly as in Fig. 2. The PD model (Eq. 4) is then used to calculate cell inhibition. For doxorubicin we use baseline lesions as well as lesions in which drug penetration therapy is applied. Experiments for dox are run both with and without the nutrient effect.

A table of average $\mathrm{IC}_{50}$ 's and $\log \left(\mathrm{IC}_{50} / \mathrm{IC}_{50 \text {,mono }}\right)$ 's for these experiments is given in Table 2. Here and throughout this paper " $\mathrm{IC}_{50 \text {, mono" }}$ "refers to baseline cells exposed in monolayer and serves as a reference. Note that, as these are simulated monolayer exposures, $\mathrm{IC}_{50 \text {,mono }}$ is deterministic. Figure 3 shows a typical nutrient profile, using Lesion II as an example with an IQR of $36 \%$. This measurement is completely analogous to that used in Fig. 2 except that here it is applied to nutrient distribution and there is no normalization since nutrient levels are bounded absolutely from 0 to 100 percent, the level within the vasculature, itself. Nutrient IQR's for the other two lesions are within $2 \%$ of this value.

At the $5 \%$ significance level, one-tailed $t$ tests show that the average $\log \mathrm{IC}_{50}$ ratio is not greater than 0 for cisplatin, underscoring the homogeneity of its distribution. In contrast, out of the four experiments performed for doxorubicin from the combinations of nutrient effect and penetration therapy, three indicate that the average log ratios are greater than 0 at the $5 \%$ significance level. Within this group of four we can analyze 
Fig. 3 Contour plot shows nutrient distribution in Lesion II demonstrating significant heterogeneity. Other lesions are similar

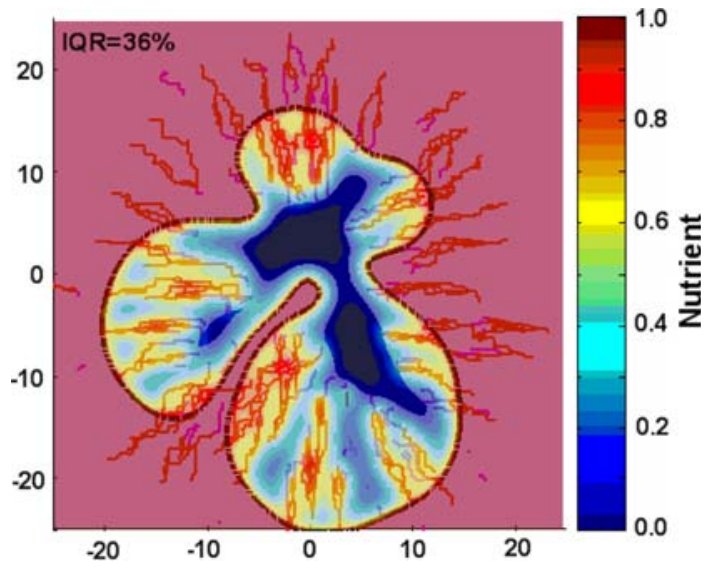

the strength of the nutrient effect. For the baseline lesion, the nutrient effect increases the $\log \mathrm{IC}_{50}$ ratio by 0.406 units (a factor of about 2.5). For the lesion with penetration therapy, the increase is 0.274 units (a factor of about 1.9). Paired $t$ tests show that these differences are significant at the $5 \%$ level.

Cell inhibition distributions closely mirror their AUC distributions, with that of cisplatin being virtually uniform at $50 \%$ inhibition throughout. Conversely, doxorubicin displays heterogeneity, increased with the addition of the nutrient effect. Using Lesion II as a representative example for doxorubicin, the upper block of frames in Fig. 4 demonstrates the inhibition distributions for the baseline lesion with and without the nutrient effect. While the broadening of the cumulative probability plot as well as a comparison of the color distribution plots indicate that the nutrient effect increases heterogeneity, inhibition IQR is reduced from 81 to $77 \%$ (again, not normalized). The effect of penetration therapy in the lower block of frames is readily apparent. IQR's, color plots, and probability graphs all indicate more uniform inhibition, ranging moderately from 35 to $65 \%$. Again we see increased heterogeneity in the plots with the addition of the nutrient effect. This time the IQR also reflects the increase. Lesions I and III yield similar results.

Third experiment In our third set of simulations, we investigate the effect of therapies designed to improve doxorubicin penetration under several combinations of drug/interstitum diffusivities, cell densities, and drug efflux activities (e.g., Pgp). Figure 5 gives bar graphs of (a) $\log \mathrm{IC}_{50}$ ratios and (b) AUC interquartile ranges for three scenarios. The leftmost triplet corresponds to baseline tumor density and no efflux, resulting in a condition of "normal" cellular uptake. The middle triplet corresponds to high density with no efflux, a condition of high uptake. The rightmost corresponds to baseline density with efflux, a condition of low uptake. For the $\log \mathrm{IC}_{50}$ ratio (a), in the baseline tumor case there is a change of -0.388 log units in going from no removal of hyaluronic acid to almost complete removal. When density is increased, the change increases to -0.709 ; however, when Pgp efflux is activated, ANOVA reveals there is no statistical difference, and in fact, the measured change is positive. Results are similar when the nutrient effect is included, with all bars essentially 

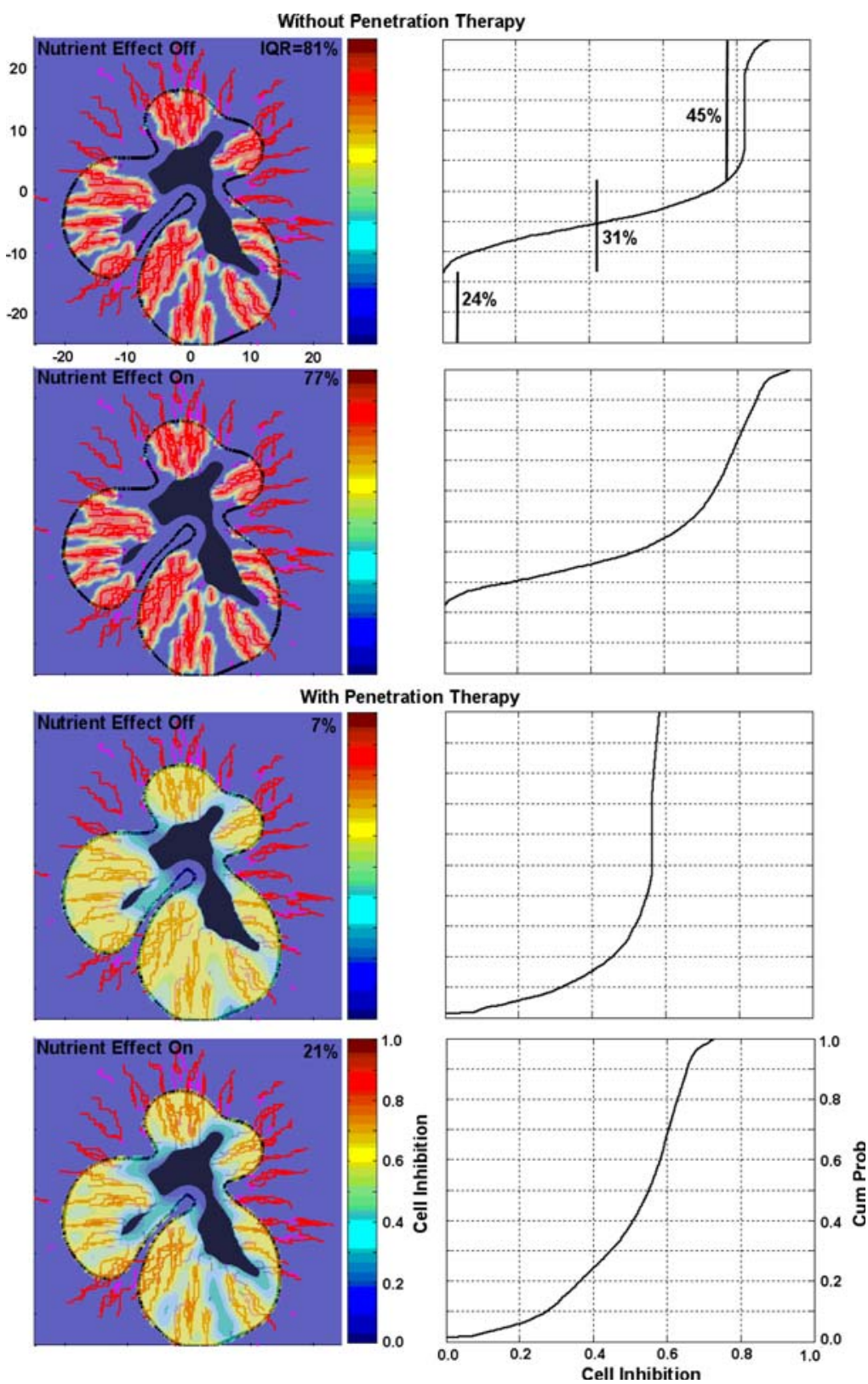

Fig. 4 (Upper block) Cell growth inhibition profile of Lesion II at baseline settings with and without the nutrient effect after bolus administration depicted in Fig. 2. Probability plot and IQR are now of inhibition distribution and are not normalized with respect to any average. Although the IQR indicates decreased heterogeneity with the nutrient effect, both the color distribution plot and the probability plot indicate increased heterogeneity as is evidenced by the broadening of the curve. (Lower block) The same experiment, except with doxorubicin penetration increased. Now both the plots and IQR show increased heterogeneity. The appropriate $\mathrm{IC}_{50}$ is used in each experiment 


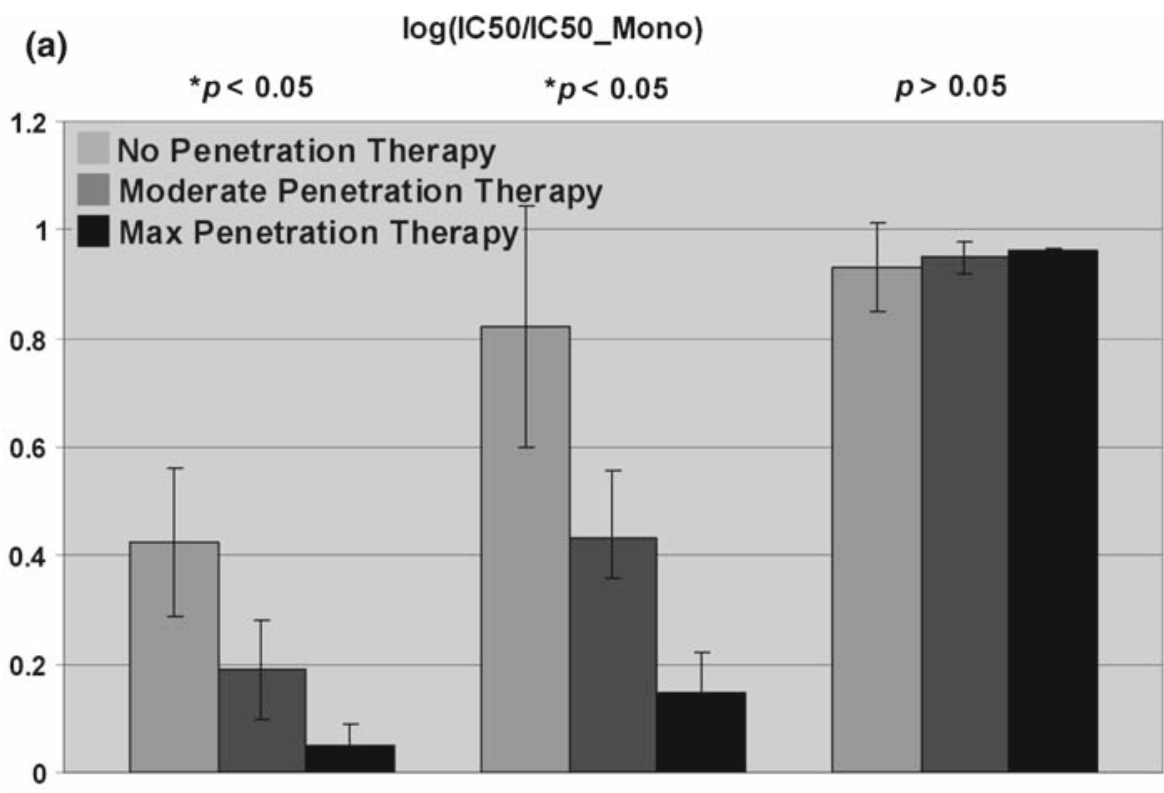

(b)

AUC Interquartile Range (\%)

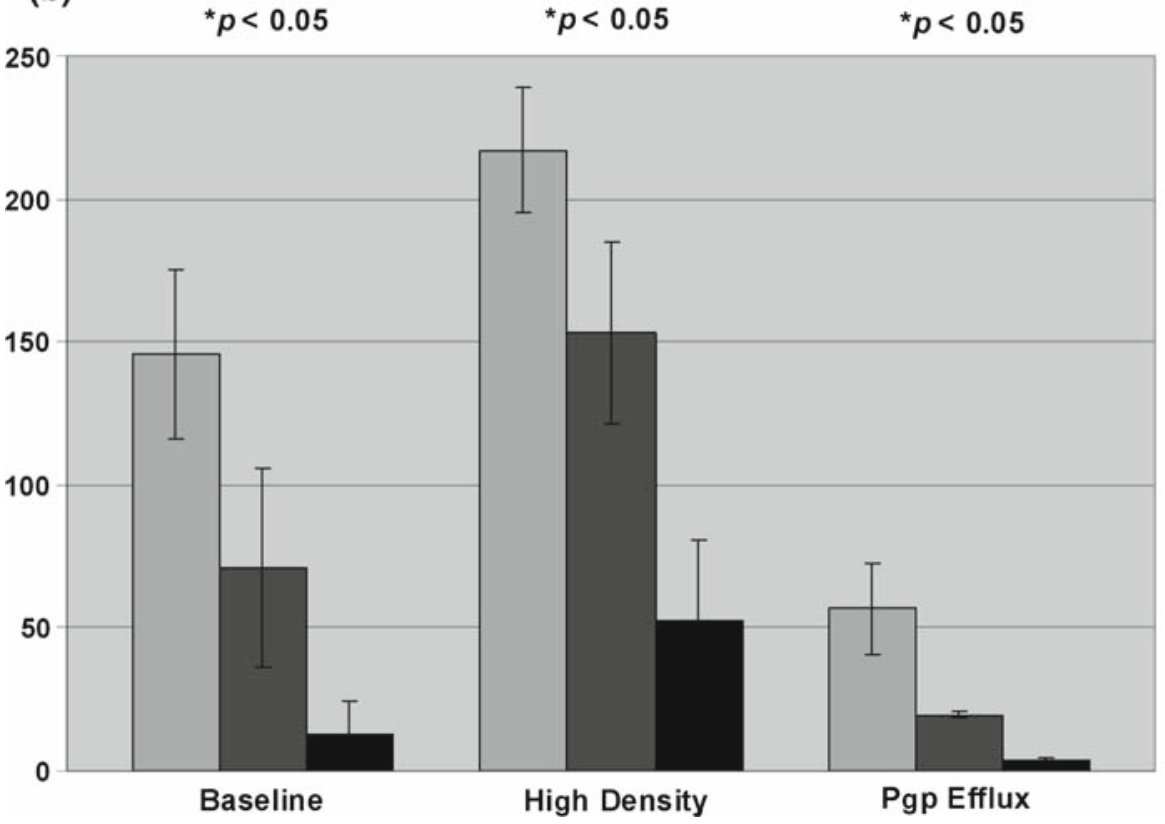

Fig. 5 The effect of increasing doxorubicin penetration on a $\log \left(\mathrm{IC}_{50} / \mathrm{IC}_{50, \text { mono }}\right)$ and $\mathbf{b}$ interquartile range shown in three cases: baseline tumor (excepting penetration therapy), high-density tumor, and normal density tumor with Pgp efflux. High density has the effect of increasing drug uptake, while Pgp efflux has the opposite effect. Three replications per bar with standard deviations and results of ANOVA displayed 


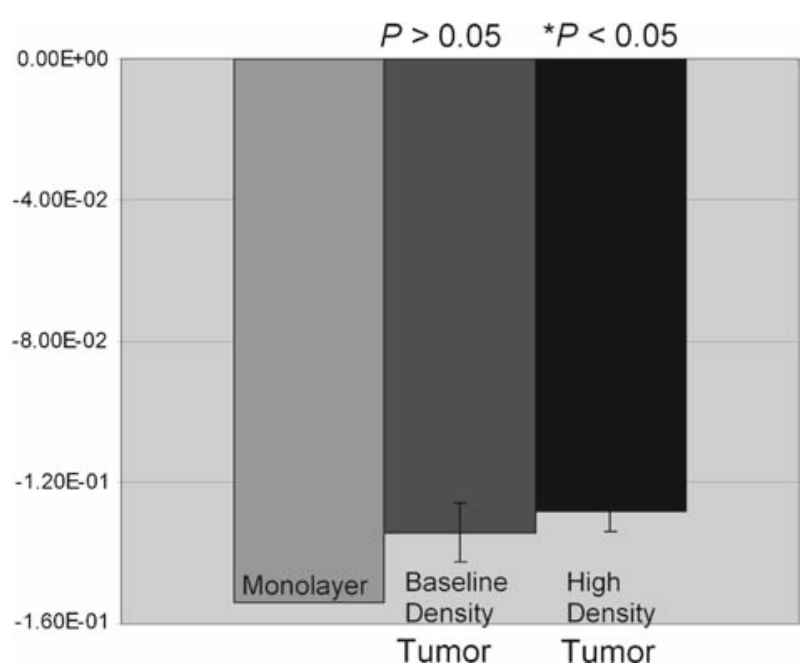

Fig. 6 Effect of permeabilization therapy with respect to cisplatin is shown in three cases. Bars are of $\log \left(\mathrm{IC}_{50, \text { perm }} / \mathrm{IC}_{50}\right.$, unperm $)$ where "perm" and "unperm" correspond to permeabilized and unpermeabilized conditions. Three replications per bar with results of two-tailed $t$ tests relative to monolayer displayed. While there is a statistical difference at the 0.05 significance level for the high-density tumor, this disappears at the 0.01 significance level

increased by a constant, approximately 0.37. For the AUC interquartile range (b), it is seen that heterogeneity is greatest in the high density case and least in the Pgp efflux case. Within each triplet the heterogeneity decreases with increasing penetration therapy, as expected. The magnitudes of change mirror those for the $\log \mathrm{IC}_{50}$ ratios, with the baseline case experiencing a moderate change (from 146 to 13\%), the high density case experiencing a dramatic change (from 217 to 52\%), and the Pgp efflux case experiencing the least change (from 57 to $4 \%$ ).

Fourth experiment In our fourth and final set of simulations we investigate the effect of permeabilization therapy vis-a-vis cisplatin. Figure 6 shows $\log \left(\mathrm{IC}_{50}\right.$,perm/ $\mathrm{IC}_{50 \text {, unperm }}$ ) for three cases: monolayer, in vivo with baseline cell density, and in vivo with high cell density. Here, the subscripts "perm" and "unperm" denote the application or withholding of permeabilization therapy. Permeabilization results in a decrease of $0.154 \log \mathrm{IC}_{50}$ units for simulated monolayers, i.e., a reduction of $\mathrm{IC}_{50}$ by a factor of 0.7, and is thus effective in vitro. An interesting question is whether this carries over in vivo, i.e., whether a monolayer assay can be used to predict clinical efficacy. Improvements for the two in vivo simulations are comparable to monolayer results, with all three log-differences about -0.14 , and no statistical difference between improvement for the baseline case and for monolayer at the $5 \%$ significance level using a two-tailed $t$ test.

\section{Discussion}

In agreement with experimental observation, our simulations show that heterogeneities of drug, nutrient, and oxygen, caused in part by irregular vasculature and lesion 
morphology, exist and can significantly impact therapeutic results. Moreover, the sense and magnitude of their influence is not always intuitively obvious. A good example of this is that, despite its well-noted penetration difficulties, doxorubicin performs well clinically. Our simulations show that this may be somewhat explained by its retention in tissue removed from vasculature, causing homogeneity of exposure to increase long after the bolus has been terminated (Fig. 2). This phenomenon has been experimentally verified in [19] with spheroids. Because of this, the resulting cell inhibition distribution is more homogenous than would otherwise be expected. On the other hand, cisplatin maintains an homogenous DNA-bound distribution at all times from bolus initiation to $20 \mathrm{~h}$ later, resulting in an extremely uniform cell inhibition distribution. This result, as well as the near equality of its $\mathrm{IC}_{50}$ and $\mathrm{IC}_{50 \text {, mono }}$ demonstrated in Table 2 , has also been experimentally verified with spheroids $[18,32,37]$.

While retention in tissue contributes to the performance of doxorubicin in vivo, Table 2 demonstrates that its heterogeneity of distribution contributes to increased serum drug concentrations to match the same cell inhibition in monolayer. In one case, the average amount of drug increases by nearly one log unit. It is reasonable to expect that heterogeneity of nutrient, resulting in hypoxia and hypoglycemia, should compound this problem for doxorubicin. Indeed, this is the case as can be seen by the approximate doubling of the $\mathrm{IC}_{50}$ 's $(0.482 \mu \mathrm{M}$ vs. 1.34 and 0.197 vs. 0.371$)$ when the nutrient effect is applied. By graphically and quantitatively showing corresponding cell inhibition distributions. Figure 4 offers further insight into these phenomena. It is easily seen that cell inhibition distributions are as heterogeneous as their corresponding DNAbound AUC distributions, with areas of lesion removed from vasculature experiencing reduced cell inhibition. An examination of the probability plots in the upper block, corresponding to baseline lesions, shows that a full $24 \%$ of viable lesion undergoes no inhibition at all. It is clear from these graphs that penetration therapy greatly decreases heterogeneity of cell inhibition (and commensurately, $\mathrm{IC}_{50}$ ) as does removal of the nutrient effect. The latter may be clinically feasible through, for example, carbogen breathing or recombinant human Epo (rHuEPO) administration [63,75].

One puzzling behavior is that while both the color and probability plots demonstrate consistently increased heterogeneity brought about by the nutrient effect (as is evidenced by the broadening of the probability curves), the IQR actually decreases in the baseline case from 81 to $77 \%$. This occurs with Lesions I and III as well. A solution to the mystery is obtained by noticing that, absent the nutrient effect, a large portion of the tumor either experiences no inhibition (about 24\%, which is distant from the vasculature) or an already heterogeneous inhibition (about 31\%). The remaining 45\% receives a near homogeneous level of inhibition (the vertical portion of the curve), and this fraction corresponds to tissue close to the vasculature. Thus, the only significant heterogeneity that can be induced by the nutrient effect is within this fraction. Indeed, it is just this part of the curve that broadens in the second probability distribution, indicating greater heterogeneity, as expected. As the IQR is designed to measure heterogeneity somewhat more globally, it misses - in fact, misdiagnoses - the change occurring within this fraction.

In addition to dosing requirements, there is a second and subtler reason to consider heterogeneity of the inhibition distribution when treating clinical tumors. Heterogeneities in microenvironmental conditions have been linked to increased lesion 
fragmentation and invasiveness [12,25,39,45,55]. While the mechanisms underlying this phenomenon are complex, involving myriad protein signaling events and activities at the cellular level, they may at least partly rely on gross lesion effects, including those caused by drug administration.

Figure 5 shows what might be expected from therapies that increase doxorubicin penetration by, for example, removing hyaluronic acid. As expected, for the baseline tumors, greater homogeneity and level of AUC is achieved, resulting in reductions of $\mathrm{IC}_{50}$. This effect has been experimentally verified using spheroids $[37,38]$. That it should be more pronounced for high-density in silico tumors and completely absent in the presence of Pgp efflux is intriguing. A potential explanation is availed by simplifying the pharmacokinetics model (Eqs. 2), reducing it to the one-dimensional, one-compartment steady state diffusion equation $0=D \nabla^{2} s-k s$ with diffusivity $D$ and uptake rate $k$. In two dimensions, a segment of blood vessel acting as a source next to a section of tissue approximates the one-dimensional case. This equation has one governing parameter, the characteristic diffusion length $L=\sqrt{D / k}$, and a (non-unique) solution $s_{\mathrm{V}} \exp (-x / L)$, where $x$ is distance from the source and $s_{\mathrm{V}}$ is the constant level of drug in the vasculature. Considering a section of tissue of thickness $d$ next to a vessel and a fixed exposure time, average AUC is proportional to the integral

$$
\int_{0}^{d} s_{\mathrm{V}} \exp (-x / L) d x=s_{\mathrm{V}} L(1-\exp (-d / L)) .
$$

Let $\mathrm{AUC}_{50}$ be the fixed average AUC required for fifty percent cell inhibition. Then, ignoring the constant of proportionality,

$$
\mathrm{AUC}_{50}=\mathrm{IC}_{50} L(1-\exp (-d / L))
$$

Increasing the diffusivity to simulate penetration therapy results in an increased characteristic length $C L$, where $C>1$, and hence a new $\mathrm{IC}_{50}$. The ratio of $\mathrm{IC}_{50}$ 's is therefore

$$
\frac{\mathrm{AUC}_{50} / C L(1-\exp (-d / C L))}{\mathrm{AUC}_{50} / L(1-\exp (-d / L))}=\frac{(1-\exp (-d / L))}{C(1-\exp (-d / C L))},
$$

which approaches 1 as $L \rightarrow \infty$, and approaches $1 / C$ as $L \rightarrow 0$. Now, increasing cell density has the effect of increasing $k$, resulting in a smaller $L$, thus manifesting the differential in $\mathrm{IC}_{50}$ 's. Conversely, activating Pgp efflux has the effect of decreasing $k$, resulting in a larger $L$, thus nullifying the differential.

That something along these lines is probably happening is demonstrated in Fig. 5b by the generally high IQR's for the high density case versus the low IQR's for the Pgp, and the large change in the high density triplet versus the Pgp triplet. Indeed, there is experimental evidence that Pgp and other transporters might facilitate drug transport further away from vasculature [77]. This could be an important point when deciding upon appropriate therapies for tumors exhibiting different characteristics such as efflux mechanisms and relatively high or low densities. Any therapy involves risk. There may 
be less to gain under certain conditions, advising that the therapy not be performed or perhaps, that concomitant therapy be performed designed to optimize conditions.

The great homogeneity of both cisplatin AUC and cell inhibition demonstrated in Fig. 2 and Table 2 indicate that in vitro assays using this drug may have relatively high positive predictive accuracy. While our simulations do not yield enough resolution to claim that the results herein answer this question, the outcome shown in Fig. 6 is of interest. There is no statistical difference at the 0.01 significance level between improvement obtained by permeabilization therapy in the simulated tumors (even very dense ones) and monolayer. This compares favorably with the findings of [72] in which the improvement obtained via cisplatin permeabilization therapy in vitro is strongly reflected by the improvement in isolated lung perfusion in rats.

While it can and should be argued that the simulations herein fail to account for some (many!) critical aspects of tumor growth and drug response (such as clonal heterogeneity, cell phase sensitivity, and signaling pathways) and that parameter settings may in some cases be inexact, it should not be concluded that these shortcomings invalidate characteristics the simulations have revealed. Indeed, we have correctly post-dicted several results: that doxorubicin retention results in a more uniform AUC and cell inhibition than would otherwise be indicated by its penetration difficulties [19]; that Pgp and other transporters might facilitate drug penetration into lesion tissue [77]; that cisplatin achieves a highly uniform AUC, and its cellular in vitro monolayer inhibition can closely match that of in vitro spheroids [18,32,37]; and that improvement due to cisplatin permeabilization therapy in vitro has the potential to predict improvement in vivo [72]. Through the model we have provided evidence that not only do macroscopic environmental conditions, namely, drug and nutrient distribution heterogeneity, potentially greatly impact therapeutic efficacy, but also that the outcome of therapeutic strategies can depend upon them in nonlinear and a priori unpredictable ways. The results of our third set of experiments (doxorubicin penetration therapy) provide an example. In light of this, it would be prudent to devote attention to factors residing at coarser and more global scales than solely the genetic.

One of our broader goals is to demonstrate how increasingly sophisticated in silico technology, driven by mathematical modeling and calibrated with experimental data, can and is being developed to provide an alternate investigative and clinical tool complementary to traditional methods $[6,24,61,62,67]$. It can well be imagined that were doxorubicin and cisplatin discovered today, the in vivo simulations herein presented could be used to anticipate their lesion- and cellular-scale pharmacokinetics, helping to refine clinical trial design and lower costs. In clinical application, the results could be used to guide therapeutic strategy. For example, any risks associated with doxorubicin penetration therapy could be minimized if it were known that the patient's tumor were expressing Pgp or otherwise had lowered cellular uptake, according to the results given in Fig. 5. With further development, we anticipate that in silico models and methods similar to those in this paper will become increasingly accurate and useful. Towards that end we are working on incorporating a more veridical model of vasculature along the lines of McDougall and Stephanou, in which blood flow plays a key role in the formation of vasculature [46,69]. We are furthermore developing 3D models, which are proving successful at accurately simulating morphological evolution [23]. One area of special interest is that of liposomal and nanovectored delivery. These have 
the potential of unprecedented accuracy and specificity of delivery [22]. It would be interesting to adapt the PKPD model herein, paying particular attention to transport in and extravasation from blood vessels [28], to see what predictions can be made.

The power of in vitro experimentation lies in its ease of implementation while remaining in the biological realm. By its very nature, in vitro experimentation attempts to refine and isolate. Yet, much of what happens in vivo is the result of a nonlinear system whose behavior is more than the sum of its parts. The power of in silico simulation lies in its ability to integrate components into a virtual system capable of reproducing such behavior, implicitly taking into account circuits of information flow difficult to explicitly analyze. Accurately calibrated and rigorously validated, such an integrated model could provide a "dry-lab" to be used as a powerful complement to the traditional wet-lab in fundamental research, drug discovery, and the clinic. It could be used to probe scenarios and test hypotheses that are either difficult or impossible to instantiate in the body. Results could then suggest supportive in vitro and in vivo experimentation, the end result being new therapeutic targets or strategies. Simultaneously, weaknesses (or strengths!) of the in silico model could be uncovered and addressed. Computational models have the potential to facilitate an era of great discovery and progress in understanding and treating cancer, and providing new hope to its victims.

Acknowledgments The authors would like to thank Steve Wise (U.C. Irvine) for assistance with numerical methods and programming, Ardith El-Kareh (U. of Arizona) for insights into the cellular pharmacokinetics and pharmacodynamics of doxorubicin and cisplatin, Hoa P. Nguyen (U.C. Irvine) for helpful discussions regarding experimental protocols and drug response, John Fruehauf (U.C. Irvine) for suggestions and corrections covering a broad spectrum of oncological knowledge, and the anonymous referees for their helpful comments.

Open Access This article is distributed under the terms of the Creative Commons Attribution Noncommercial License which permits any noncommercial use, distribution, and reproduction in any medium, provided the original author(s) and source are credited.

\section{References}

1. Alarcón T, Byrne H, Maini P (2003) A cellular automaton model for tumour growth in inhomogeneous environment. J Theor Biol 225:257-274

2. Anderson A, Chaplain M (1998) Continuous and discrete mathematical models of tumor-induced angiogenesis. Bull Math Biol 60:857-899

3. Arancia G, Calcabrini A, Meschini S, Molinari A (1998) Intracellular distribution of anthracyclines in drug resistant cells. Cytotechnology 27:95-111

4. Araujo R, McElwain D (2004) A history of the study of solid tumour growth: the contribution of mathematical modelling. Bull Math Biol 66:1039-1091

5. Baish J, Gazit Y, Berk D, Nozue M, Baxter L, Jain R (1996) Role of tumor vascular architecture in nutrient and drug delivery: an invasion percolation-based network model. Microvasc Res 51:327-346

6. Bangs A, Paterson T (2003) Finding value in in silico biology. Biosilico 1:18-22

7. Bellomo N, Prezioso L (2000) Modelling and mathematical problems related to tumor evolution and its interaction with the immune system. Math Comput Model 32:413-452

8. Breward C, Byrne H, Lewis C (2003) A multiphase model describing vascular tumour growth. Bull Math Biol 65(4):609-640

9. Chaplain M (1996) Avascular growth, angiogenesis and vascular growth in solid tumors: the mathematical modelling of the stages of tumour development. Math Comput Model 23:47-87 
10. Chen Y, Simon S (2000) In situ biochemical demonstration that P-glycoprotein is a drug efflux pump with broad specificity. J Cell Biol 148:863-870

11. Cristini V, Blawzdziewicz J, Loewenberg M (2001) An adaptive mesh algorithm for evolving surfaces: simulations of drop breakup and coalescence. J Comput Phys 168:445-463

12. Cristini V, Frieboes H, Gatenby R, Caserta S, Ferrari M, Sinek J (2005) Morphologic instability and cancer invasion. Clin Cancer Res 11:6772-6779

13. Cristini V, Lowengrub JS, Nie Q (2003) Nonlinear Simulation of Tumor Growth. J Math Biol 46(3): 191-224. doi:10.1007/s00285-002-0174-6

14. DeGregorio M, Lui G, Macher B, Wilbur J (1984) Uptake, metabolism, and cytotoxicity of doxorubicin in human Ewing's sarcoma and rhabdomyosarcoma cells. Cancer Chemother Pharmacol 12:59-63

15. Demant E, Friche E (1998) Kinetics of anthracycline accumulation in multidrug-resistant tumor cells: relationship to drug lipophilicity and serum albumin binding. Biochem Pharmacol 56:1209-1217

16. Demant E, Sehested M, Jensen P (1990) A model for computer simulation of P-glycoprotein and transmembrane delta $\mathrm{pH}$-mediated anthracycline transport in multidrug-resistant tumor cells. Biochim Biophys Acta 1055:117-125

17. Dordal M, Ho A, Jackson-Stone M, Fu Y, Goolsby C, Winter J (1995) Flow cytometric assessment of the cellular pharmacokinetics of fluorescent drugs. Cytometry 20:307-314

18. Durand R (1986) Chemosensitivity testing in V79 spheroids: drug delivery and cellular microenvironment J Natl Cancer Inst 77:247-252

19. Durand R (1990) Slow penetration of anthracyclines into spheroids and tumors: a therapeutic advantage? Cancer Chemother Pharmacol 26:198-204

20. El-Kareh A, Secomb T (2003) A mathematical model for cisplatin cellular pharmacodynamics. Neoplasia 5:161-169

21. El-Kareh A, Secomb T (2005) Two-mechanism peak concentration model for cellular pharmacodynamics of Doxorubicin. Neoplasia 7:705-713

22. Ferrari M (2005) Cancer nanotechnology: opportunities and challenges. Nat Rev Cancer 5:161-171

23. Frieboes H, Lowengrub J, Wise S, Zheng X, Macklin P, Bearer E, Cristini V (2007) Computer simulation of glioma growth and morphology. NeuroImage 37:S59-S70

24. Frieboes H, Zheng X, Sun C, Tromberg B, Gatenby R, Cristini V (2006) An integrated computational/experimental model of tumor invasion. Cancer Res 66:1597-1604

25. Frieboes HB, Sinek JP, Nalcioglu O, Fruehauf JP, Cristini V (2006) Nanotechnology in cancer drug therapy: a biocomputational approach. In: Ferrari M, Lee AP, Lee LJ (eds) BioMEMS and biomedical nanotechnology, vol I, Chap 15. Springer, New York, NY, pp 435-460

26. Fruehauf J (2002) In vitro assay-assisted treatment selection for women with breast or ovarian cancer. Endocr Relat Cancer 9:171-182

27. Fruehauf J, Bosanquet A (1993) In vitro determination of drug response: a discussion of clinical applications. Princ Pract Oncol Updates 7:1-16

28. Gentile F, Ferrari M, Decuzzi P (2007) Transport of nanoparticles in blood vessels: the effect of vessel permeability and blood rheology. Ann Biomed Eng 36:254-261

29. Gulledge C, Dewhirst M (1996) Tumor oxygenation: a matter of supply and demand. Anticancer Res 16:741-749

30. Haroon Z, Peters KG, Greenberg CS, Dewhirst MW (1999) Angiogenesis and blood flow in solid tumors. In: Teicher B (ed) Antiangiogenic agents in cancer therapy, Chap 1. Humana Press, Totowa, NJ, pp 3-21

31. Hurwitz S, Terashima M, Mizunuma N, Slapak C (1997) Vesicular anthracycline accumulation in doxorubicin-selected U-937 cells: participation of lysosomes. Blood 89:3745-3754

32. Inoue S, Holland JF, Wasserman L (1985) Susceptibility of multicellular tumor spheroids (MTS) to doxorubicin (DXR) and cisplatin. Proc Am Assoc Cancer Res 26:341

33. Jackson $\mathrm{T}$ (2003) Intracellular accumulation and mechanism of action of doxorubicin in a spatiotemporal tumor model. J Theor Biol 220:201-213

34. Jain R (2001) Delivery of molecular medicine to solid tumors: lessons from in vivo imaging of gene expression and function. J Control Release 74:7-25

35. Jekunen A, Shalinsky D, Hom D, Albright K, Heath D, Howell S (1993) Modulation of cisplatin cytotoxicity by permeabilization of the plasma membrane by digitonin in vitro. Biochem Pharmacol 45:2079-2085

36. Jiang Y, Pjesivac-Grbovic J, Cantrell C, Freyer J (2005) A multiscale model for avascular tumor growth. Biophys J 89:3884-3894 
37. Kohno N, Ohnuma T, Kaneko M, Holland J (1988) Interactions of doxorubicin and cis-platin in squamous carcinoma cells in culture. Br J Cancer 58:330-334

38. Kohno N, Ohnuma T, Truog P (1994) Effects of hyaluronidase on doxorubicin penetration into squamous carcinoma multicellular tumor spheroids and its cell lethality. J Cancer Res Clin Oncol 120:293297

39. Kunkel P, Ulbricht U, Bohlen P, Brockmann M, Fillbrandt R, Stavrou D, Westphal M, Lamszus K (2001) Inhibition of glioma angiogenesis and growth in vivo by systemic treatment with a monoclonal antibody against vascular endothelial growth factor receptor-2. Cancer Res 61:6624-6628

40. Lankelma J, Dekker H, Luque F, Luykx S, Hoekman K, van der Valk P, van Diest P, Pinedo H (1999) Doxorubicin gradients in human breast cancer. Clin Cancer Res 5:1703-1707

41. Lee A (1987) Coordinated regulation of a set of genes by glucose and calcium ionophores in mammalian cells. Trends Biochem Sci 12:20-23

42. Levasseur L, Faessel H, Slocum H, Greco W (1998) Implications for clinical pharmacodynamic studies of the statistical characterization of an in vitro antiproliferation assay. J Pharmacokinet Biopharm 26:717-733

43. Macklin P, Lowengrub J (2005) Evolving interfaces via gradients of geometry-dependent interior Poisson problems: application to tumor growth. J Comput Phys 203:191-220

44. Macklin P, Lowengrub J (2006) An improved geometry-aware curvature discretization for level set methods: application to tumor growth. J Comput Phys 215:392-401

45. Macklin P, Lowengrub J (2007) Nonlinear simulation of the effect of microenvironment on tumor growth. J Theor Biol 245:677-704

46. McDougall S, Anderson A, Chaplain M (2006) Mathematical modelling of dynamic adaptive tumour-induced angiogenesis: clinical implications and therapeutic targeting strategies. J Theor Biol 241:564-589

47. McDougall S, Anderson A, Chaplain M, Sherratt J (2002) Mathematical modelling of flow through vascular networks: implications for tumour-induced angiogenesis and chemotherapy strategies. Bull Math Biol 64:673-702

48. McGhee J, von Hippel P (1974) Theoretical aspects of DNA-protein interactions: co-operative and non-co-operative binding of large ligands to a one-dimensional homogeneous lattice. J Mol Biol $86: 469-489$

49. Mellor H, Ferguson D, Callaghan R (2005) A model of quiescent tumour microregions for evaluating multicellular resistance to chemotherapeutic drugs. Br J Cancer 93:302-309

50. Minchinton A, Tannock I (2006) Drug penetration in solid tumors. Nat Rev Cancer 6:583-592

51. Mueller-Klieser W (1984) Microelectrode measurement of oxygen tension distributions in multicellular spheroids cultured in spinner flasks. Recent Results Cancer Res 95:134-149

52. Norris ES, King JR, Byrne HM (2006) Modelling the response of spatially structured tumours to chemotherapy: drug kinetics. Math Comput Model 43:820-837

53. Nugent L, Jain R (1984) Extravascular diffusion in normal and neoplastic tissues. Cancer Res $44: 238-244$

54. Padera T, Stoll B, Tooredman J, Capen D, di Tomaso E, Jain R (2004) Pathology: cancer cells compress intratumour vessels. Nature 427:695

55. Pennacchietti S, Michieli P, Galluzzo M, Mazzone M, Giordano S, Comoglio P (2003) Hypoxia promotes invasive growth by transcriptional activation of the met protooncogene. Cancer Cell 3:347-361

56. Please CP, Pettet G, McElwain DLS (1998) A new approach to modelling the formation of necrotic regions in tumours. Appl Math Lett 11:89-94

57. Primeau A, Rendon A, Hedley D, Lilge L, Tannock I (2005) The distribution of the anticancer drug doxorubicin in relation to blood vessels in solid tumors. Clin Cancer Res 11:8782-8788

58. Qu X, Wan C, Becker H, Zhong D, Zewail A (2001) The anticancer drug-DNA complex: femtosecond primary dynamics for anthracycline antibiotics function. Proc Natl Acad Sci USA 98:14212-14217

59. Rizzo V, Sacchi N, Menozzi M (1989) Kinetic studies of anthracycline-DNA interaction by fluorescence stopped flow confirm a complex association mechanism. Biochemistry 28:274-282

60. Sadowitz P, Hubbard B, Dabrowiak J, Goodisman J, Tacka K, Aktas M, Cunningham M, Dubowy R, Souid A (2002) Kinetics of cisplatin binding to cellular DNA and modulations by thiol-blocking agents and thiol drugs. Drug Metab Dispos 30:183-190

61. Sanga S, Frieboes H, Zheng X, Gatenby R, Bearer E, Cristini V (2007) Predictive oncology: a review of multidisciplinary, multiscale in silico modeling linking phenotype, morphology and growth. Neuroimage 37(Suppl 1):S120-134 
62. Sanga S, Sinek J, Frieboes H, Ferrari M, Fruehauf J, Cristini V (2006) Mathematical modeling of cancer progression and response to chemotherapy. Expert Rev Anticancer Ther 6:1361-1376

63. Shannon A, Bouchier-Hayes D, Condron C, Toomey D (2003) Tumour hypoxia, chemotherapeutic resistance and hypoxia-related therapies. Cancer Treat Rev 29:297-307

64. Shen J, Subjeck J, Lock R, Ross W (1989) Depletion of topoisomerase II in isolated nuclei during a glucose-regulated stress response. Mol Cell Biol 9:3284-3291

65. Simon S, Schindler M (1994) Cell biological mechanisms of multidrug resistance in tumors. Proc Natl Acad Sci USA 91:3497-3504

66. Sinek J, Frieboes H, Zheng X, Cristini V (2004) Two-dimensional chemotherapy simulations demonstrate fundamental transport and tumor response limitations involving nanoparticles. Biomed Microdevices 6:297-309

67. Sinek JP, Frieboes HB, Sivaraman B, Sanga S, Cristini V (2006) Mathematical and computational modeling: towards the development of nanodevices for drug delivery. In: Kumar, Challa SSR (eds) Nanotechnologies for the life sciences vol 4: Nanodevices for the life sciences, Chap 2. Wiley, Germany, pp 29-66

68. Siu W, Arooz T, Poon R (1999) Differential responses of proliferating versus quiescent cells to adriamycin. Exp Cell Res 250:131-141

69. Stéphanou A, McDougall S, Anderson A, Chaplain M (2006) Mathematical modelling of the influence of blood rheological properties upon adaptive tumour-induced angiogenesis. Math Comput Model 44:96-123

70. Swabb E, Wei J, Gullino P (1974) Diffusion and convection in normal and neoplastic tissues. Cancer Res 34:2814-2822

71. Takemura Y, Kobayashi H, Miyachi H, Hayashi K, Sekiguchi S, Ohnuma T (1991) The influence of tumor cell density on cellular accumulation of doxorubicin or cisplatin in vitro. Cancer Chemother Pharmacol 27:417-422

72. Tanaka T, Kaneda Y, Li T, Matsuoka T, Zempo N, Esato K (2001) Digitonin enhances the antitumor effect of cisplatin during isolated lung perfusion. Ann Thorac Surg 72:1173-1178

73. Tannock I, Lee C, Tunggal J, Cowan D, Egorin M (2002) Limited penetration of anticancer drugs through tumor tissue: a potential cause of resistance of solid tumors to chemotherapy. Clin Cancer Res 8:878-884

74. Tarasiuk J, Frézard F, Garnier-Suillerot A, Gattegno L (1989) Anthracycline incorporation in human lymphocytes. Kinetics of uptake and nuclear concentration. Biochim Biophys Acta 1013:109-117

75. Teicher BA, Herman TS, Holden SA, Wang YY, Pfeffer MR, Crawford JW, Frei E (1990) Tumor resistance to alkylating agents conferred by mechanisms operative only in vivo. Science $247: 1457-$ 1461

76. Troger V, Fischel J, Formento P, Gioanni J, Milano G (1992) Effects of prolonged exposure to cisplatin on cytotoxicity and intracellular drug concentration. Eur J Cancer 28:82-86

77. Tunggal J, Melo T, Ballinger J, Tannock I (2000) The influence of expression of p-glycoprotein on the penetration of anticancer drugs through multicellular layers. Int J Cancer 86:101-107

78. Tyson R, Stern L, LeVeque R (2000) Fractional step methods applied to a chemotaxis model. J Math Biol 41:455-475

79. Wang D, Lippard S (2005) Cellular processing of platinum anticancer drugs. Nat Rev Drug Discov 4:307-320

80. Ward J, King J (2003) Mathematical modelling of drug transport in tumour multicell spheroids and monolayer cultures. Math Biosci 181:177-207

81. Zheng J, Chen C, Au J, Wientjes M (2001) Time- and concentration-dependent penetration of doxorubicin in prostate tumors. AAPS PharmSci 3:E15

82. Zheng X, Wise S, Cristini V (2005) Nonlinear simulation of tumor necrosis, neo-vascularization and tissue invasion via an adaptive finite-element/level-set method. Bull Math Biol 67:211-259 